\title{
Minderheiten: Anerkennung - Toleranz und Förderung - Mehrwert
}

Gibt es einen erfassbaren Mehrwert für die Mehrheitsbevölkerung durch ihr Zusammenleben mit Minderheiten? [...] Welchen Nutzen haben Mehrheitsbevölkerungen von der positiven Diskriminierung, welchen Nachteil von ihrer negativen Diskriminierung?

fragt Matthias Theodor Vogt (ua) in EJM 2-2009¹ und ruft dabei „Sprach-, Kultur-, Politik-, Wirtschafts-, Rechts- und Naturwissenschaftler [...] zu einer Analyse“ auf, „wer von einer positiven Diskriminierung von Minderheiten profitiert.“ Dabei sollte, so Vogt, der „Vorteil für alle“, also auch jener für die betreffenden Mehrheiten, in den Mittelpunkt wissenschaftlicher Untersuchungen rücken.

Ein klares „Ja“ auf die Frage nach dem „Vorteil für alle“ oder dem „Mehrwert durch Minderheiten“ antworten zwei neuere und insofern epochale Europarat-Dokumente, nämlich die Entschließung 301 (2010) und die Empfehlung 286 (2010) des Kongresses der Gemeinden und Regionen, die unter dem Titel „Minderheitensprachen - ein wertvolles Gut für die Regionalentwicklung“ stehen. ${ }^{2}$ Dabei ist neben dem kulturellen vor allem auch der wirtschaftliche Mehrwert ins Zentrum (auch) des politischen Interesses gerückt. Ein ökonomischer Mehrwert kann gemäß dem die beiden Dokumente argumentativ untermauernden Erläuternden Bericht dann - und nur dann - erzielt werden, wenn bestimmte Grundbedingungen des fördernden Minderheitenschutzes (der positiven Diskriminierung oder affirmative action) ${ }^{3}$ erfüllt sind, wobei dies im Idealfall auch Formen der regionalen Selbstverwaltung ${ }^{4}$ sowie einen regionalen oder lokalen Amtssprachenstatus der betreffenden Minderheitensprache(n) - oder zumindest die Möglichkeit der Verwendung auch der Minderheitensprache(n) im Behördenverkehr - umfassen sollte.

\footnotetext{
${ }^{1}$ Mehrwert durch Minderheiten? Das VIII. Collegium Pontes Görlitz-Zgorzelec-Zhořelec.

2 Mit einer Einführung vollinhaltlich abgedruckt in EJM 2-2010.

3 Zur Anerkennung dieses Prinzips auf der EU-Ebene vgl Waldemar Hummer, EJM 1-2011.

4 Zum 2009 beim Europarat erarbeiteten „Bezugsrahmen für regionale Demokratie“ vgl EJM 2-2010.
} 
Auch Vladimir Kreck greift diesen Ansatz auf und widmet sich in seinem Beitrag der ökonomischen Seite eines möglichen Mehrwerts, der sich - unter bestimmten Voraussetzungen - aus der Existenz autochthoner Minderheiten für die betreffenden Regionen ergeben kann. Kreck erläutert dabei einige wichtige Anhaltspunkte, die für einen direkten Zusammenhang zwischen kultureller Vielfalt und ökonomischer Prosperität sprechen, nicht ohne zugleich weitergehende Forschungsfragen aufzuwerfen.

$\mathrm{Zu}$ den „Vorbedingungen für positive ökonomische Aspekte“ zählt Kreck neben bestimmten „Strukturbedingungen“ im Zeichen des Prinzips der positiven Diskriminierung der betreffenden Minderheiten auch den „Grad an Toleranz und Konfliktbewältigung, den Minderheit(en) und Mehrheit gemeinsam erreicht haben“.

Wie aber kann die Toleranz zwischen Minderheit(en) und Mehrheit erreicht bzw begünstigt werden? Sicher ist, dass die gezielte Förderung einer autochthonen Minderheit mit dem Ziel der Wahrung ihrer ethnisch-kulturellen Identität geeignet ist, auf Seiten der Minderheitsangehörigen Ängste abzubauen und insofern prinzipiell toleranzfördernd wirken kann. Eine Minderheitenförderung im Sinne eines ,von oben“, dh primär politisch und rechtlich verordneten Minderheitenschutzes dürfte jedoch ohne eine breite Akzeptanz auch auf Seiten der Mehrheitsbevölkerung nicht dauerhaft lebensfähig sein.

In der Praxis hat es sich jedenfalls immer wieder als zielführend erwiesen, in Fragen des Minderheitenschutzes nicht nur auf der politischen, sondern auch auf der gesellschaftlichen Ebene zu verhandeln, dies konkret durch Einbeziehung von Vertretern sowohl der Minderheits- als auch der Mehrheitsbevölkerung - wobei gerade hier die Perspektive des Vorteils für alle von entscheidender Bedeutung ist. Dabei hat es sich auch gezeigt, dass gegenseitige Akzeptanz bzw Toleranz nicht,,von heute auf morgen“" erreicht werden, sondern nur langsam, im Rahmen stetiger Begegnung und auch Auseinandersetzung (zB im Rahmen der Aufarbeitung gemeinsamer bzw trennender Geschichte) wachsen können.

In Südtirol beispielsweise dauerte es allein zwanzig Jahre, bis nach dem Inkrafttreten des zweiten Autonomiestatuts 1972 fast sämtliche dazugehörigen Durchführungsbestimmungen verabschiedet wurden, worauf auch der betreffende Streitfall zwischen Österreich und Italien vor der UNO im Jahr 1992 für beigelegt erklärt werden konnte. Dazwischen lag eine Phase akribischen Ausverhandelns von Kompromissen, in welches über paritätische Kommissionen ganz bewusst nicht nur Vertreter des Staates, sondern auch der deutschen Minderheit und der italienischen Mehrheit vor Ort miteinbezogen worden waren..$^{5}$ Auf diese Weise konnte die Akzeptanz des Südtiroler Minderheitenschutz-Reglements auch auf der gesellschaftlichen Ebene weitgehend gewährleistet werden - wobei dessen schrittweise Umsetzung nicht zufällig von einem wirtschaftlichen Aufschwung begleitet war, in dessen Gefolge Südtirol bis heute beeindruckende Wirtschaftsdaten aufweist.

Einen langwierigen Konfliktfall hatte auch Südtirols Schutzstaat (kin-state) Österreich zu bewältigen. Zwar betraf der sog Kärntner Ortstafelstreit nur einen, aber zweifellos keinen unbedeutenden Teilaspekt der durch den österreichischen Staatsvertrag von 1955 (ua) für die slowenische Minderheit in Kärnten gewährleisteten Rechte.

\footnotetext{
${ }^{5}$ Zum Thema Südtirol vgl die Beiträge von Franz Matscher in EJM 1-2008 und von Walter Siegl in EJM 3/4-2010. 
Während die Rechte auf Verwendung des Slowenischen als Amtssprache und auf slowenischen Elementarunterricht wesentlich früher im Rechtssystem Österreichs und des Bundeslandes Kärnten verankert werden konnten, gab es in der Frage, wie hoch der Anteil slowenischsprachiger Kärntner in einer Ortschaft sein müsse, um eine Pflicht zur Aufstellung zweisprachiger Ortstafeln ${ }^{6}$ nach sich zu ziehen, über lange Zeiträume hinweg weder einen politischen noch einen gesellschaftlichen Konsens. Daran konnte weder die im Volksgruppengesetz von 1976 festgelegte (und niemals vollständig umgesetzte) $25 \%$-Grenze noch die vom österreichischen Verfassungsgerichtshof 2001 befürwortete 10\%-Marke etwas ändern. Die daraus sich ergebende rechtsstaatliche Brisanz - Nichtumsetzung des betreffenden Verfassungsgerichtsurteils von 2001 und der entsprechenden Folgejudikatur - hat Martin Hiesel bereits in EJM 3/4-2010 ausführlich erläutert. In diesem Heft geht Hiesel nun auf den im Frühjahr/Sommer 2011 erzielten Kompromiss in der Ortstafelfrage ein. Dabei bemängelt er zwar, dass der Kompromiss ,nur um den Preis des partiellen Unterlaufens einer volksgruppenfreundlichen Regelung“ durch das oberste Verfassungsgericht Österreichs ,erkauft“ werden konnte, räumt aber andererseits ein, dass auf diese Weise eine „Verfassungsrechtslage geschaffen“ werden konnte, „der offenkundig weitgehende Akzeptanz [Hervorhebung d Verf] zukommt“. Ein Blick auf die Vorgeschichte des Kärntner Kompromisses legt offen, dass diesem ein zähes Ringen aller politisch und gesellschaftlich maßgeblich Beteiligten, konkret der Bürgermeister der betroffenen Gemeinden, der politischen Parteien, der Heimatverbände und der Organisationen der Kärntner Slowenen um eine konsensfähige Lösung vorangegangen ist. Somit scheint sich auch anhand des Konfliktes um die Kärntner Ortstafeln zu bestätigen, dass (Minderheiten-)Recht im Zweifel nur dann dauerhaft lebensfähig ist, wenn es nicht nur „von oben“ verordnet, sondern auch durch einen im Laufe der Zeit und im Rahmen stetiger Begegnung und Überzeugungsarbeit gewachsenen gesellschaftlichen Mindestkonsens „,von unten“ abgefedert wird.

Zwar ist der Kärntner Kompromiss noch jung und muss sich demgemäß erst bewähren, dies sollte die Wissenschaft aber nicht davon abhalten, gerade aus diesem Anlass den historischen Verlauf des Ortstafelkonfliktes zu analysieren - nicht zuletzt auch, um den mit Minderheitenfragen in Theorie und Praxis Befassten weitere Anhaltspunkte für mögliche Stolpersteine und Erfolgsfaktoren auf dem Weg zur Lösung eines Minderheitenkonfliktes zu liefern. In diesem Sinne widmet sich Claudia Fräss-Ehrfeld in ihrem Beitrag vor allem den Problemfaktoren, die aus ihrer Sicht eine Lösung des Kärntner Ortstafelkonflikts über einen längeren Zeitraum hinweg verhindert hatten.

Während die Kärntner Slowenen bereits im Zuge des österreichischen Staatsvertrages von 1955 und innerstaatlich durch das Volksgruppengesetz von 1976 grundsätzlich als nationale Minderheit (in Österreich „Volksgruppe“") anerkannt und demgemäß auch problemlos in den Schutzbereich des Europarat-Rahmenübereinkommens zum Schutz nationaler Minderheiten einbezogen wurden, steht eine solche Anerkennung der Kornen, deren Kultur und autochthone Sprache ebenso wie jene der gälisch spre-

\footnotetext{
${ }^{6}$ Zum kulturellen und sprachlichen Aspekt historisch gewachsener Ortsnamen vgl Heinz-Dieter Pohl: Ortsnamen in einer historisch gewachsenen Kulturlandschaft unter besonderer Berücksichtigung Kärntens und Osttirols in EJM 2-2009.
} 
chenden Einwohner von Schottland, Wales und Nordirland großteils auf keltischen Wurzeln basiert, noch aus. Der vom Cornwall Council, der Verwaltungsbehörde für Cornwall initiierte und von Ian Saltern verfasste Cornish National Minority Report 2 enthält ein argumentativ fundiertes Plädoyer zugunsten der Anerkennung der Kornen als „,nationale Minderheit“ im Sinne des Europarat-Rahmenübereinkommens. Aus europäischer Perspektive ist dabei bemerkenswert, dass das Rahmenübereinkommen trotz fehlender eigener Minderheitendefinition bestimmten nach Anerkennung strebenden Minderheiten argumentative Hilfestellung zu bieten vermag. In der kurzen redaktionellen Einführung zum Minority Report 2 wird erläutert, dass dies vor allem der rechtsstaatlich fundierten Auslegungsarbeit des an der Umsetzung des Rahmenübereinkommens beteiligten Expertengremiums, des sog Beratenden Ausschusses, zu verdanken ist.

Welchen Nutzen haben Mehrheitsbevölkerungen von der positiven Diskriminierung, welchen Nachteil von ihrer negativen Diskriminierung?

Diese von Matthias Theodor Vogt gestellte Frage könnte auch im Falle Cornwalls interessant werden, bietet doch womöglich auch hier die Anerkennung und Förderung einer historisch gewachsenen kulturell-sprachlichen Minderheit Chancen und Vorteile für alle Beteiligten.

Beate Sibylle Pfeil 